\title{
Reproductive Toxicity of Pomegranate Peel Extract Synthesized Gold Nanoparticles: A Multigeneration Study in C. elegans
}

\author{
Mahnoor Patel $\left(\mathbb{D},{ }^{1}\right.$ Nikhat J. Siddiqi $\left(\mathbb{D},{ }^{2}\right.$ Preeti Sharma, ${ }^{1}$ Abdullah S. Alhomida $\left(\mathbb{D},{ }^{2}\right.$ \\ and Haseeb A. Khan $\mathbb{D}^{2}$ \\ ${ }^{1}$ Department of Biotechnology, Veer Narmad South Gujarat University, 395007, Surat, Gujarat, India \\ ${ }^{2}$ Department of Biochemistry, College of Science, King Saud University, Riyadh 11451, Saudi Arabia \\ Correspondence should be addressed to Haseeb A. Khan; khan_haseeb@yahoo.com
}

Received 28 April 2019; Revised 30 June 2019; Accepted 11 July 2019; Published 28 August 2019

Guest Editor: Ziyou Zhou

Copyright (C) 2019 Mahnoor Patel et al. This is an open access article distributed under the Creative Commons Attribution License, which permits unrestricted use, distribution, and reproduction in any medium, provided the original work is properly cited.

\begin{abstract}
C. elegans is a preferential model for testing environmental toxicity of compounds including nanomaterials. The impact of multigeneration exposure of gold nanoparticles (AuNPs) on the lifespan and fertility of C. elegans is not known and therefore is investigated in this study. We used pomegranate (Punica granatum) peel extracts as a reducing agent to synthesize gold nanoparticles (PPE-AuNPs) from chloroauric acid. Nematodes were grown till adult stage and then exposed to 25, 50, and $100 \mu \mathrm{g} / \mathrm{ml}$ of PPE-AuNPs at $20^{\circ} \mathrm{C}$ for 72 hours and then assessed for lifespan and fertility. The same protocols were followed for subsequent F1, F2, and F3 generations. The results showed that PPE-AuNPs dose-dependently but insignificantly reduced the lifespan of C. elegans. Exposure of PPE-AuNPs significantly and dose-dependently reduced the fertility of $C$. elegans in terms of the number of eggs produced. The reproductive toxicity of PPE-AuNPs was found to be minimal in parental generation (F0) and maximal in F3 generation. In conclusion, biologically synthesized PPE-AuNPs adversely affect the fertility of C. elegans while the factors responsible for reproductive toxicity are inherited by subsequent generations.
\end{abstract}

\section{Introduction}

There is a growing trend for the synthesis and widespread use of nanoparticles (NPs) for applications in different areas such as medicine, engineering, bioremediation, cosmetics, and food industry [1-7]. Such a wider scope of engineered NPs needs to be channelized by their prudent use for availing their benefits without ignoring their adverse effects on humans and the environment [8]. The extensive use of nanoparticle has led to their release in the environment causing them to be considered environmental toxicants [9]. Among the metallic NPs, gold nanoparticles (AuNPs) have been recognized as promising tools for biomedical applications due to their biocompatibility and ease in functionalization $[10,11]$. Although gold is a chemically inert material, its transformation into nanoscale imparts the antigenic quality as several studies have shown an acute phase immune activation following injection of AuNPs in rats [12-15] and mice [16, 17]. However, the primed animals (preexposed to AuNPs) showed protection against AuNP-induced acute immune activation in the form of reduced expression of proinflammatory cytokines [18].

Caenorhabditis elegans (C. elegans) is a free-living, transparent nematode with a short life cycle of a few days and can be quickly grown in a laboratory. Interestingly, the majority of human genes have homologs in C. elegans. A comparative proteomics study has shown that $83 \%$ of the worm proteome possesses human homologous genes [19]. C. elegans is therefore considered a model organism for understanding human gene function. The neural system, immune system, and digestive and reproductive systems of C. elegans are identical to the vertebrates which makes it an excellent model for toxicity studies as well [20-22]. C. elegans is ideally suited for the screening of NPs while mitigating the hurdles associated with the use of mammalian animal models, such as ethical issues, handling expertise, space requirement, and time constraints [23-25]. 
The pomegranate (Punica granatum) is a fruit-bearing deciduous shrub belonging to the family Lythraceae. Pomegranate fruits possess antioxidant and medicinal properties and are commonly used in baking, cooking, juice blends, meal garnishes, smoothies, and alcoholic beverages. Recently, pomegranate juice and peel extracts have been utilized as reducing and stabilizing agents for the synthesis of AuNPs [26-29]. Kim et al. [30] have shown that parental (F0) exposure of $10 \mathrm{~nm}$ size AuNPs caused reproductive toxicity in unexposed F2 generation which was gradually recovered in the unexposed F3 and F4 generations. We hypothesized that the antioxidant potential of pomegranate peel extract [31] would modify the toxicological profile of AuNPs synthesized from it. In this investigation, we studied the transgenerational effects of pomegranate peel extract synthesized gold nanoparticles (PPE-AuNPs) on the lifespan and fertility of $C$. elegans after exposing parental (F0) and three generations (F1, F2, and F3) of C. elegans to three different concentrations of PPE-AuNPs.

\section{Materials and Methods}

2.1. Synthesis of PPE-AuNPs. Pomegranate fruits were purchased from the local market. The fresh peel $(50 \mathrm{~g})$ obtained from a prewashed pomegranate fruit was added to $250 \mathrm{ml}$ of distilled water and boiled for $25 \mathrm{~min}$. The same ratio of pomegranate peel and solvent has been reported earlier for obtaining the extract [31]. The extract was filtered using a Whatman filter paper to get the aqueous fruit peel extract. The freshly prepared extract was stored in a sterile bottle and kept at $4^{\circ} \mathrm{C}$ for a short time to cool the extract. Pomegranate peel extract $(0.9 \mathrm{ml})$ was mixed with $25 \mathrm{ml}$ of $1 \mathrm{mM}$ solution of chloroauric acid $\left(\mathrm{HAuCl}_{4}\right)$. The reacting mixture was kept for $24 \mathrm{~h}$ at room temperature with intermittent shaking. The color change from gold to pink indicated the synthesis of PPE-AuNPs. The synthesized nanoparticles were washed with sterile water using the sequential steps of centrifugation $(17,000 \mathrm{~g}, 30 \mathrm{~min})$, supernatant removal, and redispersion by vortex. Aqueous dispersions of PPE-AuNPs in the concentrations of 10,50 , and $100 \mu \mathrm{g} / \mathrm{ml}$ were used for C. elegans exposure.

2.2. Characterization of PPE-AuNPs. UV-visible spectrophotometry (Shimadzu, Japan) was used to monitor the bioreduction of $\mathrm{AuCl}_{4}{ }^{-}$ions to metallic Au leading to the synthesis of PPE-AuNPs. The wavelength range from $300 \mathrm{~nm}$ to $800 \mathrm{~nm}$ was scanned for obtaining the absorption spectra of PPE-AuNPs. Scanning electron microscopy (SEM, Jeol, Japan) was used to characterize the morphology and size of the synthesized PPE-AuNPs. An energy-dispersive X-ray spectrometer (EDX, Oxford System) was used for elemental analysis. A sample for EDX analysis was prepared by sprinkling the dispersed PPE-AuNPs onto a double-sided adhesive carbon conductive tape which was then mounted on the microscopic copper stub. Photographic images were visualized on a computer screen, and the results of SEM and EDX were interpreted.
2.3. Maintenance of Caenorhabditis elegans. C. elegans (wild type, Bristol, N2) were obtained from the National Institute of Immunology, New Delhi, India. All worms were cultured in Nematode Growth Media (NGM: NaCl, $3 \mathrm{~g} / \mathrm{l}$; peptone, $2.5 \mathrm{~g} / \mathrm{l}$; agar, $17 \mathrm{~g} / \mathrm{l} ; 1 \mathrm{M}$ potassium phosphate, $25 \mathrm{ml} ; 1 \mathrm{M} \mathrm{CaCl}_{2}, 1 \mathrm{ml} ; 1 \mathrm{M} \mathrm{MgSO}$, $1 \mathrm{ml}$; and cholesterol, $1 \mathrm{ml}$ ) on agar plates. The food source was Escherichia coli OP50 strain. To obtain synchronized worms, gravid hermaphrodites were treated with sodium hypochlorite $(5 \% \mathrm{NaOCl} / 1 \mathrm{~N} \mathrm{NaOH}, 2: 5)$ for 10 minutes and $1 \mathrm{ml}$ aliquots of the solution were centrifuged at $2000 \mathrm{rpm}$ for $2 \mathrm{~min}$ and washed with $\mathrm{K}$-medium which consisted of $0.032 \mathrm{M} \mathrm{KCl}$ and $0.051 \mathrm{M} \mathrm{NaCl}$ [32]. C. elegans were allowed to mature till the fourth larval stage for further experiments. The experimental design for multigenerational toxicity assay is summarized in Figure 1.

2.4. Lifespan Assay. Worms were grown on NGM agar till they became gravid adults. The gravid adults were bleached to obtain the eggs. All the worms were grown till adult stage, which were then exposed to 25, 50, and $100 \mu \mathrm{g} / \mathrm{ml} \mathrm{PPE-}$ AuNPs at $20^{\circ} \mathrm{C}$ for $72 \mathrm{~h}$. The young adults were then treated with fluorodeoxyuridine to inhibit the development of the next progeny. The numbers of worms were similar in control and experimental plates. Scoring was done on every alternate day for live and dead worms till all the worms died. Live and dead worms were distinguished by a touch-provoke method under a dissecting microscope with the help of worm pick. Lifespan assay was performed for every generation for a multigenerational toxicity study. The lifespan of the worm was the time between the egg and the death of the worm. The experiments were conducted in three replicates.

2.5. Fertility Assay. Age-synchronized adult worms (L4 stage/P0 worms) were exposed to different concentrations $(25,50$, and $100 \mu \mathrm{g} / \mathrm{ml})$ of PPE-AuNPs at $20^{\circ} \mathrm{C}$ for $72 \mathrm{~h}$. After exposure, the worms were washed with M9 buffer and a single worm was transferred to a $35 \mathrm{~mm}$ petri plate containing NGM medium, supplemented with E. coli OP50. The worms were washed and pelleted, and the progeny were counted under the dissecting microscope. For each test concentration and control, the numbers of eggs were counted for every single worm. Fertility assay was performed for every subsequent generation to study multigenerational toxicity. The experiments were conducted in three replicates.

The L4 stage worms were considered parent generation (F0). Parental worms were exposed to different concentrations of PPE-AuNPs. For reproductive toxicity studies, one exposed worm was transferred to a $35 \mathrm{~mm}$ petri dish to determine the brood size. The rest of the worms were transferred to $60 \mathrm{~mm}$ MGM plates to obtain the eggs by an alkali bleach method. The worms were collected and washed thrice with distilled water. The washed worms were placed in NGM plates, and the next generation was produced. The secondgeneration worms were grown till the L4 stage to produce the first filial generation (F1) which were exposed to the same concentration of PPE-AuNPs and for the same duration as conducted in F0. A single worm was isolated for reproductive 


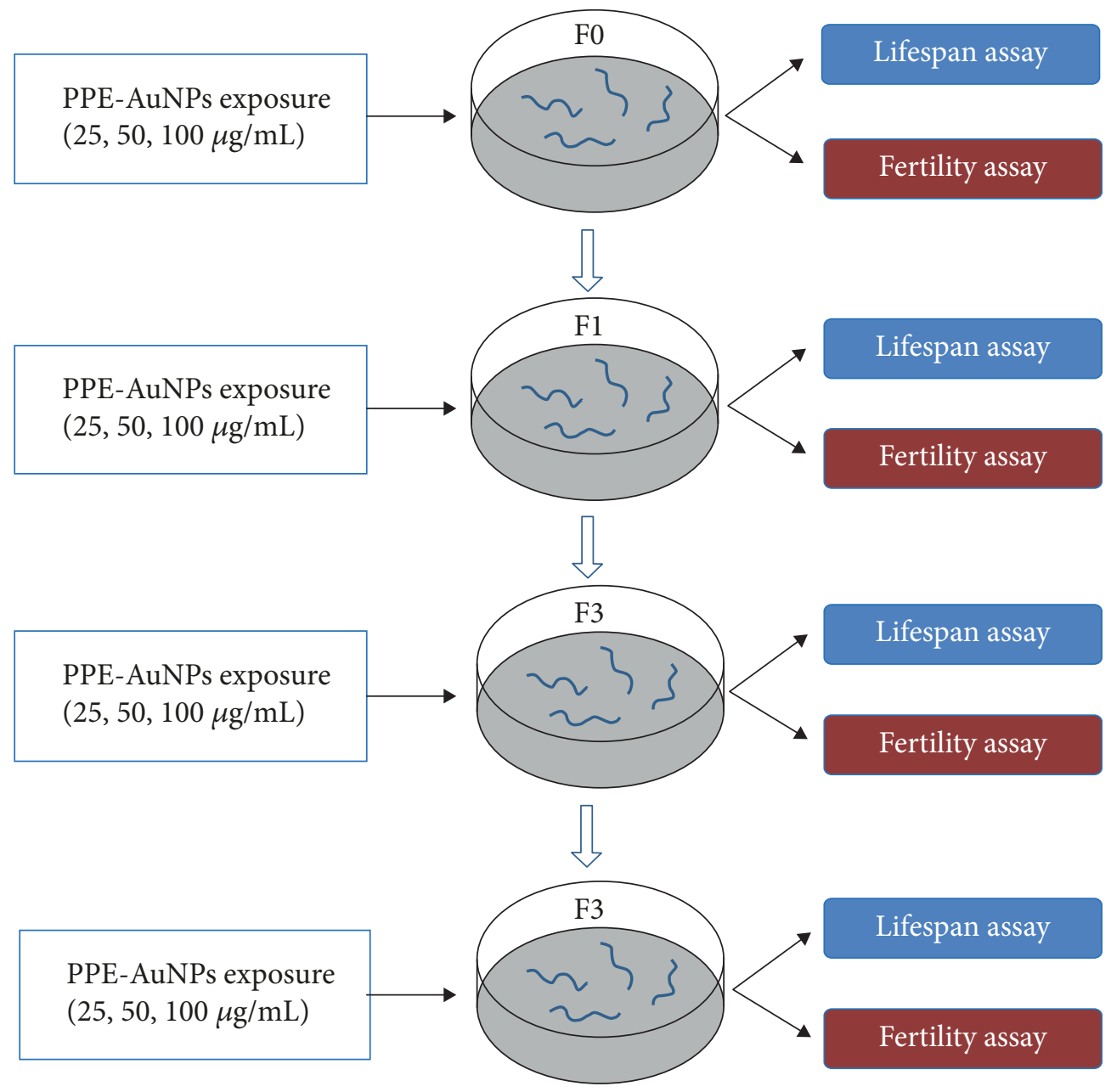

FIGURE 1: Schematic representation of study protocol for testing the effect of PPE-AuNPs on lifespan and fertility in C. elegans.

toxicity studies while the rest of the worms were allowed to grow till the L4 stage to produce the second filial generation (F2). The same protocol was followed for the third filial generation (F3).

2.6. Statistical Analysis. The data were analyzed by one-way analysis of variance (ANOVA) followed by Tukey's test using SPSS package. $P$ values $<0.05$ were considered statistically significant.

\section{Results}

3.1. Synthesis and Characterization of PPE-AuNPs. The appearance of pink color following the mixing of pomegranate extract with the solution of chloroauric acid indicated the formation of PPE-AuNPs. The UV-visible spectra of the reaction mixture showed the absorption peak at $540 \mathrm{~nm}$ with a maximal intensity at $24 \mathrm{~h}$, indicating the time course of PPE-AuNP synthesis (Figure 2(a)). The Energy Dispersive X-ray (EDX) analysis of PPE-AuNPs showed the presence of the elemental peaks of carbon, oxygen, magnesium, and gold (Figure 2(b)). The presence of peak at $2 \mathrm{keV}$ on spectrum confirmed the formation of gold nanoparticles. Small amounts of other elements including carbon, oxygen, and magnesium also appeared due to their occurrence in plant extract (Figure 2(b)). SEM image showed the morphology of PPE-AuNPs to be round with an average particle diameter of $11.0 \pm 1.5 \mathrm{~nm}$ (Figure 2(c)).

3.2. Multigenerational Effect of PPE-AuNPs on C. elegans Lifespan. The average lifespan of unexposed $C$. elegans in the parental generation (F0) was 17 days which was dosedependently reduced after exposure of PPE-AuNPs; however, this decrease in lifespan was statistically insignificant (Figure 3). Similar trends were observed in subsequent F1, F2, and F3 generations while the exposure of different concentrations of PPE-AuNPs did not cause any significant change in the lifespan of C. elegans (Figure 3).

3.3. Multigenerational Effect of PPE-AuNPs on C. elegans Fertility. The fertility assay in terms of the number of eggs showed that the parental generation (F0) unexposed C. elegans laid $195 \pm 5$ eggs which were significantly reduced following the exposure of $100 \mu \mathrm{g} / \mathrm{ml}$ PPE-AuNPs (Figure 4). Although the low $(10 \mu \mathrm{g} / \mathrm{ml})$ and medium $(50 \mu \mathrm{g} / \mathrm{ml})$ concentrations of PPE-AuNPs slightly reduced the fertility of F0 nematodes, this effect was statistically nonsignificant. However, all the concentrations of PPE- 


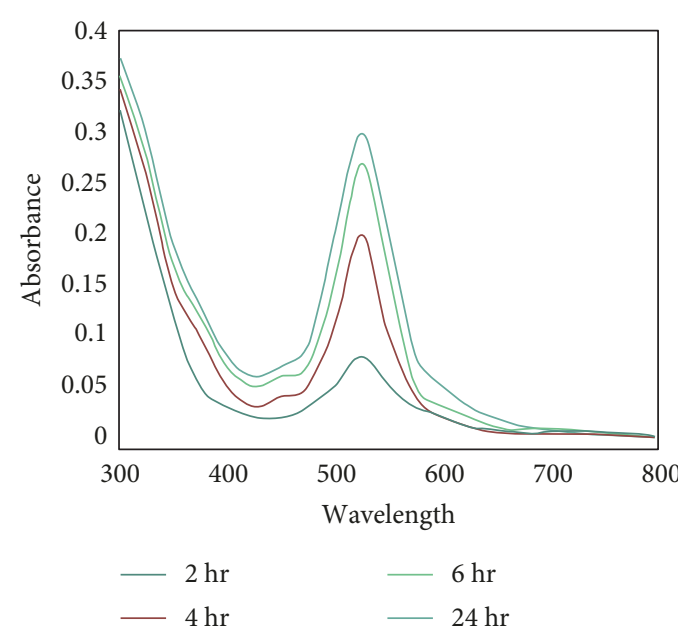

(a)

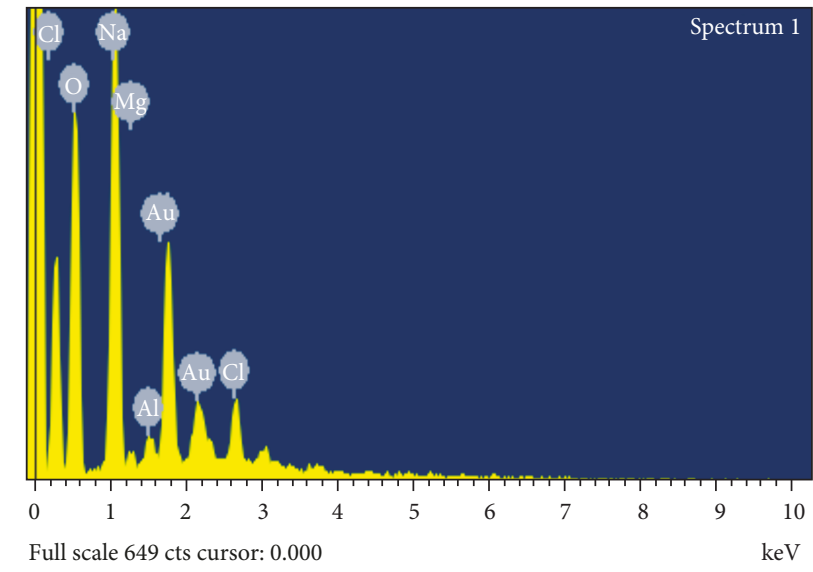

(b)

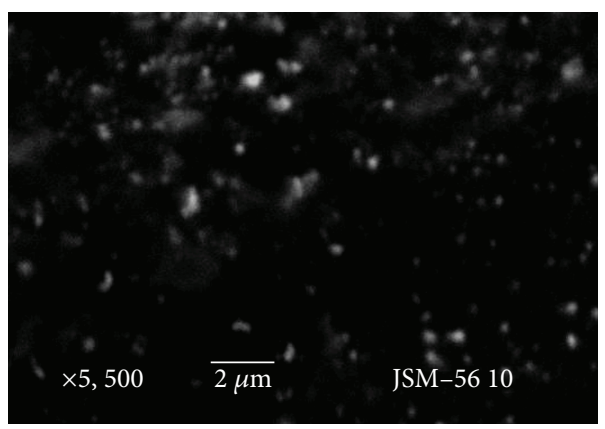

(c)

FIgURe 2: Characterization of PPE-AuNPs: (a) UV-visible spectra showing the increasing yield of PPE-AuNPs with time. (b) EDX analysis of PPE-AuNPs. (c) SEM image showing the morphology and size of PPE-AuNPs.

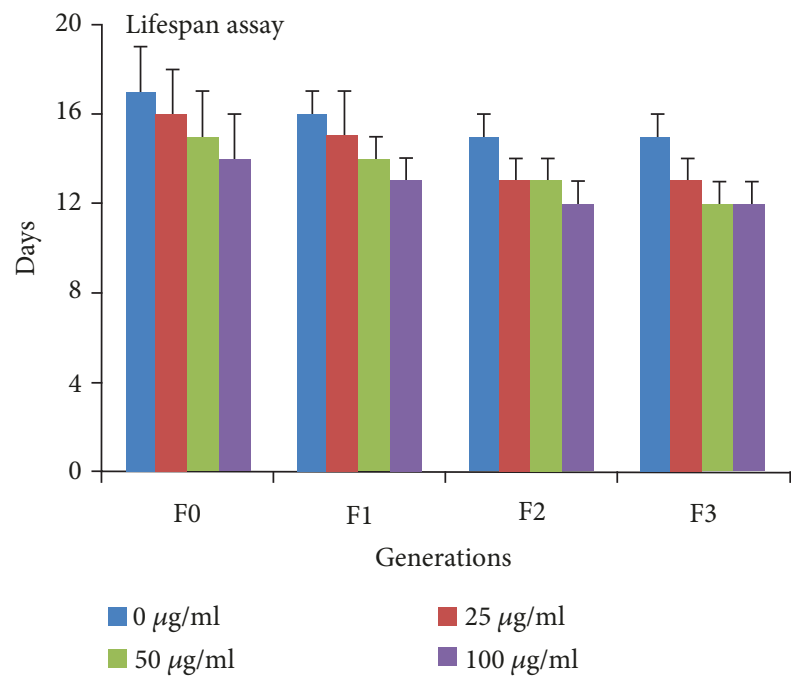

FIGURE 3: Multigenerational effect of different doses of PPE-AuNPs on the lifespan of C. elegans.

AuNPs significantly reduced the fertility of $C$. elegans in the F2 and F3 generations (Figure 4). The severity of reproductive toxicity of PPE-AuNPs in C. elegans was directly related to generations, while F0 nematodes were the least sensitive, and F3 nematodes were highly sensitive to the adverse effects of PPE-AuNPs on the fertility of $C$. elegans (Figure 4).

\section{Discussion}

In this study, we used pomegranate (Punica granatum) peel extract for the synthesis of AuNPs using a "green synthesis" approach. The production of AuNPs involves the reduction of a solution of chloroaurate ions $\left(\mathrm{AuCl}_{4}^{-}\right)$with a reducing agent. Sodium borohydride is commonly used as a reducing agent in the synthesis of AuNPs; however, it is an environmental contaminant and highly toxic to biological systems [33]. Therefore, phytochemicals like epigallocatechin [34] and mangiferin [35] have been utilized for the green synthesis of AuNPs. The reducing agent used in this study was the pomegranate peel extract (PPE) to synthesize AuNPs. Earlier studies have demonstrated the presence of gallic acid in PPE, which results in the formation of larger AuNPs when compared to other plant extracts [27]. Pomegranate extract contains ellagic acid which is responsible for its antioxidant properties. Other phenolic compounds like anthocynanins enhance the antioxidant properties of ellagic acid. The presence of the hydroxyl and carboxyl groups in plant organic 


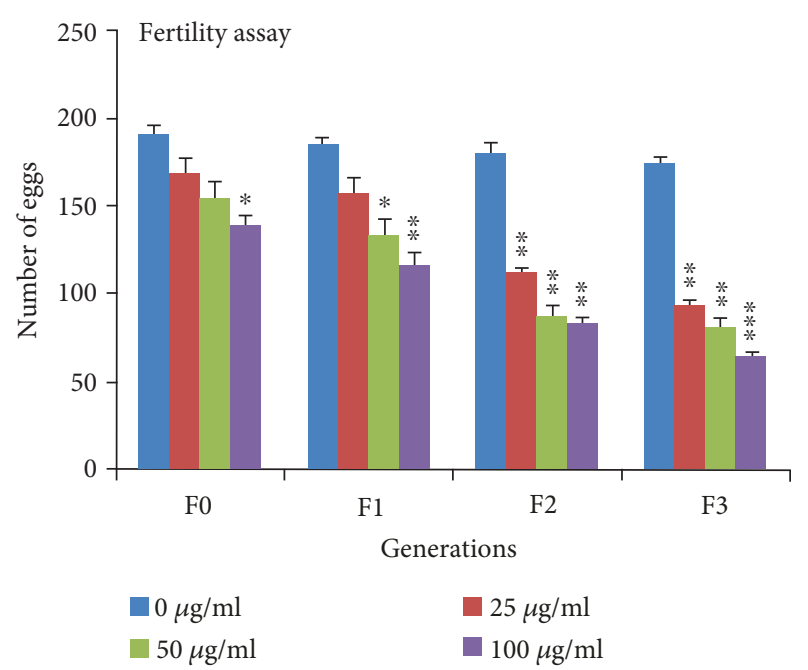

Figure 4: Multigenerational effect of different doses of PPE-AuNPs on the fertility of $C$. elegans. ${ }^{*} P<0.05,{ }^{* *} P<0.01$, and ${ }^{* * *} P<0.001$ versus the control $(0 \mu \mathrm{g} / \mathrm{ml})$ group.

compounds helps them to chelate metal ions and act as antioxidants. The interaction of metal salts with ellagic acid found in the peel extract causes esterification of the carboxyl and hydroxyl groups of ellagic acid. This leads to the loss of hydrogen from the orthophenolic hydroxyl group resulting in a semiquinone structure. Therefore, due to its easier electron-losing ability, ellagic acid can easily form $\mathrm{H}^{+}$radical which reduces gold salt to AuNPs [36].

The concentrations of PPE-AuNPs used in this study $(10-100 \mu \mathrm{g} / \mathrm{ml})$ did not demonstrate any antibacterial activity against E. coli, which was used as food for C. elegans. Elia et al. [27] have shown that AuNPs synthesized using plant extract did not exhibit any measurable adverse effect on the growth of intestinal L-cells. The minimum inhibitory concentrations (MIC) of PPE-AuNPs against various pathogenic bacteria have been reported as follows: C. albicans $(310 \mu \mathrm{g} / \mathrm{ml})$, A. flavus $(340 \mu \mathrm{g} / \mathrm{ml})$, S. aureus $(330 \mu \mathrm{g} / \mathrm{ml})$, S. typhi $(370 \mu \mathrm{g} / \mathrm{ml})$, and $V$. cholera $(410 \mu \mathrm{g} / \mathrm{ml})$ [28].

The exposure of PPE-AuNPs to C. elegans had no significant effect on the lifespan of the nematode in the parental as well as three subsequent generations studied (Figure 3). Kim et al. [30] have also demonstrated that feeding of C. elegans with E. coli dosed with AuNPs did not show any significant effect on the survival of nematodes. On the other hand, a previous study has reported toxicogenomic effects of AuNPs on C. elegans by activating some biological pathways [37]. The cellular uptake and toxicity of nanoparticles mainly depend on their size, shape, and surface chemistry [38-40]. Wang et al. [41] used the C. elegans model to understand whether the "nanoscale" size is the determining factor for the nanomaterials to induce autophagy. They used four types of similar sized nanomaterials including silicon nanoparticles (SiNP), CdTe quantum dots, carbon dots, and AuNPs. They observed that unlike other nanomaterials tested, no autophagosome formation was detected in C. elegans with SiNP exposure, suggesting that the "nanoscale" size is not the determining factor for the nanomaterials to induce autophagy in C. elegans [41]. A recent microarray study showed that developmental changes in C. elegans following mercaptoundecanoic acid-coated AuNPs were related to differential gene expression in clec174 (involved in cellular defense), cut-3 and fil-1 (both involved in body morphogenesis), dpy-14 (expressed in embryonic neurons), and mtl-1 (functions in metal detoxification and homeostasis) genes [42].

The most striking finding of this study is the reproductive toxicity of PPE-AuNPs that showed dose-dependent as well as transgeneration cumulative effects (Figure 4). Kim et al. [30] were the first to conduct a multigenerational study of AuNPs in C. elegans and revealed that reproduction rate was clearly affected in the F2 generation but then gradually recovered in the F3 and F4 generations, suggesting that just one exposure to the F0 generation can induce the adverse effects on subsequent generations. Moon et al. [43] demonstrated that continuous AuNP exposure to C. elegans impaired reproduction from F2 to F4, whereas intermittent exposure caused more pronounced effects on F3 worms, which may have resulted from damage during the convalescence period up through F2. It has been shown earlier that nanoparticles, which are able to permeate the gonad, can be transferred to the next generation [44]. Gold nanoparticles functionalized with lectin found in the tubers of the Winter Aconite (Eranthis hyemalis) plant delayed the onset of reproduction and reduced fecundity for L4 stage, preadult C. elegans [45].

Gonzalez-Moragas et al. [25] observed that citratestabilized $11 \mathrm{~nm}$ AuNPs $(100 \mu \mathrm{g} / \mathrm{ml})$ induced a significant decrease in the survival as well as reproductive output as compared to control C. elegans. However, the decreases in survival and brood size were not statistically significant in case of larger $(150 \mathrm{~nm})$ AuNPs. Other investigators also reported that smaller size nanoparticles $(<10 \mathrm{~nm})$ were more toxic to C. elegans than their larger counterparts $(>50 \mathrm{~nm})$ $[46,47]$. The higher toxicity of small sized AuNPs was attributed to their higher uptake as the worms ingested smaller AuNPs about 500 times more than the larger AuNPs [25]. The above literature indicates that small size AuNPs are more toxic for the survival of C. elegans [25] whereas the PPEAuNPs of the same size and in the same dosage are comparatively safe (Figure 3 ).

In conclusion, exposure of $11 \mathrm{~nm}$ PPE-AuNPs did not cause any significant change in the lifespan of C. elegans; however, it significantly and dose-dependently impaired the fertility of nematodes. The reproductive toxicity of PPE-AuNPs in C. elegans was found to increase in subsequent generations in the following order: $\mathrm{F} 0<\mathrm{F} 1<\mathrm{F} 2<\mathrm{F} 3$. The transgeneration flow of toxicity may be attributed to the transfer of abnormal germ cells from one generation to another. The findings of this study are of particular relevance for understanding the safety and biocompatibility of AuNPs, as strict regulatory guidelines and high cost restrict the use of laboratory animals, especially during the pilot and initial screening studies. C. elegans could serve as a model organism for testing the reproductive toxicity of many other toxicants including a wide range of nanomaterials. 


\section{Data Availability}

The data used to support the findings of this study are available from the corresponding author upon request.

\section{Conflicts of Interest}

The author(s) declare(s) that they have no conflicts of interest.

\section{Acknowledgments}

We are grateful to Dr. Arnab Mukhopadhyay and Syed Shams Tabrez from Molecular Aging Lab, National Institute of Immunology, New Delhi, for their suggestions while conducting the experiments on C. elegans. The authors would like to extend their sincere appreciation to the Deanship of Scientific Research at King Saud University for funding Research Group No. RGP-009.

\section{References}

[1] A. Weir, P. Westerhoff, L. Fabricius, K. Hristovski, and N. von Goetz, "Titanium dioxide nanoparticles in food and personal care products," Environmental Science \& Technology, vol. 46, no. 4, pp. 2242-2250, 2012.

[2] K. A. Eswar, J. Rouhi, F. S. Husairi et al., "Hydrothermal growth of flower-like $\mathrm{ZnO}$ nanostructures on porous silicon substrate," Journal of Molecular Structure, vol. 1074, pp. 140143, 2014.

[3] S. H. Kang, M. Nafiujjaman, M. Nurunnabi et al., "Hybrid photoactive nanomaterial composed of gold nanoparticles, pheophorbide-A and hyaluronic acid as a targeted bimodal phototherapy," Macromolecular Research, vol. 23, no. 5, pp. 474-484, 2015.

[4] Z. Khatun, M. Nurunnabi, M. Nafiujjaman et al., "A hyaluronic acid nanogel for photo-chemo theranostics of lung cancer with simultaneous light-responsive controlled release of doxorubicin," Nanoscale, vol. 7, no. 24, pp. 10680-10689, 2015.

[5] M. Nafiujjaman, H. A. Khan, and Y. K. Lee, "Peptide-influenced graphene quantum dots on iron oxide nanoparticles for dual imaging of lung cancer cells," Journal of Nanoscience and Nanotechnology, vol. 17, no. 3, pp. 1704-1711, 2017.

[6] M. Nafiujjaman, M. Nurunnabi, S. H. Kang, G. R. Reeck, H. A. Khan, and Y. K. Lee, "Ternary graphene quantum dot-polydopamine- $\mathrm{Mn}_{3} \mathrm{O}_{4}$ nanoparticles for optical imaging guided photodynamic therapy and $T_{1}$-weighted magnetic resonance imaging," Journal of Materials Chemistry B, vol. 3, no. 28, pp. 5815-5823, 2015.

[7] M. Nurunnabi, K. Parvez, M. Nafiujjaman et al., "Bioapplication of graphene oxide derivatives: drug/gene delivery, imaging, polymeric modification, toxicology, therapeutics and challenges," RSC Advances, vol. 5, no. 52, pp. 42141-42161, 2015.

[8] H. A. Khan and R. Shanker, "Toxicity of nanomaterials," BioMed Research International, vol. 2015, Article ID 521014, 2 pages, 2015.

[9] P. Khare, M. Sonane, Y. Nagar et al., "Size dependent toxicity of zinc oxide nano-particles in soil nematode Caenorhabditis elegans," Nanotoxicology, vol. 9, no. 4, pp. 423-432, 2015.
[10] N. Elahi, M. Kamali, and M. H. Baghersad, "Recent biomedical applications of gold nanoparticles: a review," Talanta, vol. 184, pp. 537-556, 2018.

[11] P. M. Tiwari, K. Vig, V. A. Dennis, and S. R. Singh, "Functionalized gold nanoparticles and their biomedical applications," Nanomaterials, vol. 1, no. 1, pp. 31-63, 2011.

[12] H. A. Khan, M. A. K. Abdelhalim, A. S. Alhomida, and M. S. al-Ayed, "Effects of naked gold nanoparticles on proinflammatory cytokines mRNA expression in rat liver and kidney," BioMed Research International, vol. 2013, Article ID 590730, 6 pages, 2013.

[13] H. A. Khan, K. E. Ibrahim, A. Khan, S. H. Alrokayan, and A. S. Alhomida, "Immunostaining of proinflammatory cytokines in renal cortex and medulla of rats exposed to gold nanoparticles," Histology and Histopathology, vol. 32, no. 6, pp. 597-607, 2017.

[14] H. A. Khan, K. E. Ibrahim, A. Khan, S. H. Alrokayan, A. S. Alhomida, and Y. K. Lee, "Comparative evaluation of immunohistochemistry and real-time PCR for measuring proinflammatory cytokines gene expression in livers of rats treated with gold nanoparticles," Experimental and Toxicologic Pathology, vol. 68, no. 7, pp. 381-390, 2016.

[15] H. A. Khan, M. A. K. Abdelhalim, A. S. Alhomida, and M. S. al Ayed, "Transient increase in IL- $1 \beta$, IL- 6 and TNF- $\alpha$ gene expression in rat liver exposed to gold nanoparticles," Genetics and Molecular Research, vol. 12, no. 4, pp. 5851-5857, 2013.

[16] K. E. Ibrahim, M. G. Al-Mutary, A. O. Bakhiet, and H. A. Khan, "Histopathology of the liver, kidney, and spleen of mice exposed to gold nanoparticles," Molecules, vol. 23, no. 8, p. $1848,2018$.

[17] H. A. Khan, S. Alamery, K. E. Ibrahim et al., "Size and timedependent induction of proinflammatory cytokines expression in brains of mice treated with gold nanoparticles," Saudi Journal of Biological Sciences, vol. 26, no. 3, pp. 625-631, 2019.

[18] K. E. Ibrahim, A. O. Bakhiet, M. E. Awadalla, and H. A. Khan, "A priming dose protects against gold nanoparticles-induced proinflammatory cytokines mRNA expression in mice," Nanomedicine (London, England), vol. 13, no. 3, pp. 313-323, 2018.

[19] C. H. Lai, C. Y. Chou, L. Y. Ch'ang, C. S. Liu, and W. Lin, "Identification of novel human genes evolutionarily conserved in Caenorhabditis elegans by comparative proteomics," Genome Research, vol. 10, no. 5, pp. 703-713, 2000.

[20] R. D. Handy, G. Cornelis, T. Fernandes et al., "Ecotoxicity test methods for engineered nanomaterials: practical experiences and recommendations from the bench," Environmental Toxicology and Chemistry, vol. 31, no. 1, pp. 15-31, 2012.

[21] P. R. Hunt, "The C. elegans model in toxicity testing," Journal of Applied Toxicology, vol. 37, no. 1, pp. 50-59, 2017.

[22] E. K. Marsh and R. C. May, "Caenorhabditis elegans, a model organism for investigating immunity," Applied and Environmental Microbiology, vol. 78, no. 7, pp. 2075-2081, 2012.

[23] T. Wu, H. Xu, X. Liang, and M. Tang, "Caenorhabditis elegans as a complete model organism for biosafety assessments of nanoparticles," Chemosphere, vol. 221, pp. 708-726, 2019.

[24] L. Gonzalez-Moragas, A. Roig, and A. Laromaine, "C. elegans as a tool for in vivo nanoparticle assessment," Advances in Colloid and Interface Science, vol. 219, pp. 10-26, 2015.

[25] L. Gonzalez-Moragas, P. Berto, C. Vilches et al., "In vivo testing of gold nanoparticles using the Caenorhabditis elegans model organism," Acta Biomaterialia, vol. 53, pp. 598-609, 2017. 
[26] J. Gubitosa, V. Rizzi, A. Lopedota et al., "One pot environmental friendly synthesis of gold nanoparticles using Punica granatum juice: a novel antioxidant agent for future dermatological and cosmetic applications," Journal of Colloid and Interface Science, vol. 521, pp. 50-61, 2018.

[27] P. Elia, R. Zach, S. Hazan, S. Kolusheva, Z. Porat, and Y. Zeiri, "Green synthesis of gold nanoparticles using plant extracts as reducing agents," International Journal of Nanomedicine, vol. 9, pp. 4007-4021, 2014.

[28] S. Lokina, R. Suresh, K. Giribabu, A. Stephen, R. Lakshmi Sundaram, and V. Narayanan, "Spectroscopic investigations, antimicrobial, and cytotoxic activity of green synthesized gold nanoparticles," Spectrochimica Acta Part A: Molecular and Biomolecular Spectroscopy, vol. 129, pp. 484-490, 2014.

[29] M. Ganeshkumar, M. Sathishkumar, T. Ponrasu, M. G. Dinesh, and L. Suguna, "Spontaneous ultra fast synthesis of gold nanoparticles using Punica granatum for cancer targeted drug delivery," Colloids and Surfaces B: Biointerfaces, vol. 106, pp. 208-216, 2013.

[30] S. W. Kim, J. I. Kwak, and Y. J. An, "Multigenerational study of gold nanoparticles in Caenorhabditis elegans: transgenerational effect of maternal exposure," Environmental Science \& Technology, vol. 47, no. 10, pp. 5393-5399, 2013.

[31] S. Malviya, Arvind, A. Jha, and N. Hettiarachchy, "Antioxidant and antibacterial potential of pomegranate peel extracts," Journal of Food Science and Technology, vol. 51, no. 12, pp. 41324137, 2014.

[32] P. L. Williams and D. B. Dusenbery, "Aquatic toxicity testing using the nematode, Caenorhabditis elegans," Environmental Toxicology and Chemistry, vol. 9, no. 10, pp. 1285-1290, 1990.

[33] L. Freitas de Freitas, G. Varca, J. dos Santos Batista, and A. Benévolo Lugão, "An overview of the synthesis of gold nanoparticles using radiation technologies," Nanomaterials, vol. 8, no. 11, p. 939, 2018.

[34] S. K. Nune, N. Chanda, R. Shukla et al., "Green nanotechnology from tea: phytochemicals in tea as building blocks for production of biocompatible gold nanoparticles," Journal of Materials Chemistry, vol. 19, no. 19, pp. 2912-2920, 2009.

[35] A. Y. Al-Yasiri, M. Khoobchandani, C. S. Cutler et al., "Mangiferin functionalized radioactive gold nanoparticles (MGF ${ }^{198}$ AuNPs) in prostate tumor therapy: green nanotechnology for production, in vivo tumor retention and evaluation of therapeutic efficacy," Dalton Transactions, vol. 46, no. 42, pp. 14561-14571, 2017.

[36] N. Ahmad, S. Sharma, and R. Rai, "Rapid green synthesis of silver and gold nanoparticles using peels of Punica granatum," Advanced Materials Letters, vol. 3, no. 5, pp. 376-380, 2012.

[37] O. V. Tsyusko, J. M. Unrine, D. Spurgeon et al., "Toxicogenomic responses of the model organism Caenorhabditis elegans to gold nanoparticles," Environmental Science \& Technology, vol. 46, no. 7, pp. 4115-4124, 2012.

[38] X. Ma, Y. Wu, S. Jin et al., "Gold nanoparticles induce autophagosome accumulation through size-dependent nanoparticle uptake and lysosome impairment," ACS Nano, vol. 5, no. 11, pp. 8629-8639, 2011.

[39] S. Jin, X. Ma, H. Ma et al., "Surface chemistry-mediated penetration and gold nanorod thermotherapy in multicellular tumor spheroids," Nanoscale, vol. 5, no. 1, pp. 143-146, 2013.
[40] A. Albanese, P. S. Tang, and W. C. W. Chan, "The effect of nanoparticle size, shape, and surface chemistry on biological systems," Annual Review of Biomedical Engineering, vol. 14, no. 1, pp. 1-16, 2012.

[41] Q. Wang, Y. Zhou, R. Fu et al., "Distinct autophagy-inducing abilities of similar-sized nanoparticles in cell culture and live C. elegans," Nanoscale, vol. 10, no. 48, pp. 23059-23069, 2018.

[42] C. C. Hu, G. H. Wu, S. F. Lai et al., "Toxic effects of sizetunable gold nanoparticles on Caenorhabditis elegans development and gene regulation," Scientific Reports, vol. 8, no. 1, article 15245, 2018.

[43] J. Moon, J. I. Kwak, S. W. Kim, and Y. J. An, "Multigenerational effects of gold nanoparticles in Caenorhabditis elegans: continuous versus intermittent exposures," Environmental Pollution, vol. 220, Part A, pp. 46-52, 2017.

[44] N. Mohan, C. S. Chen, H. H. Hsieh, Y. C. Wu, and H. C. Chang, "In vivo imaging and toxicity assessments of fluorescent nanodiamonds in Caenorhabditis elegans," Nano Letters, vol. 10, no. 9, pp. 3692-3699, 2010.

[45] J. Djafari, M. T. McConnell, H. M. Santos et al., "Synthesis of gold functionalised nanoparticles with the Eranthis hyemalis lectin and preliminary toxicological studies on Caenorhabditis elegans," Materials, vol. 11, no. 8, p. 1363, 2018.

[46] Q. Wu, W. Wang, Y. Li et al., "Small sizes of $\mathrm{TiO}_{2}-\mathrm{NPs}$ exhibit adverse effects at predicted environmental relevant concentrations on nematodes in a modified chronic toxicity assay system," Journal of Hazardous Materials, vol. 243, pp. 161-168, 2012.

[47] S. Gupta, T. Kushwah, A. Vishwakarma, and S. Yadav, "Optimization of ZnO-NPs to investigate their safe application by assessing their effect on soil nematode Caenorhabditis elegans," Nanoscale Research Letters, vol. 10, no. 1, 2015. 


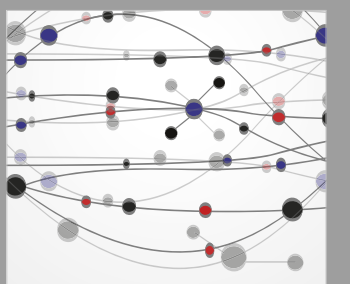

The Scientific World Journal
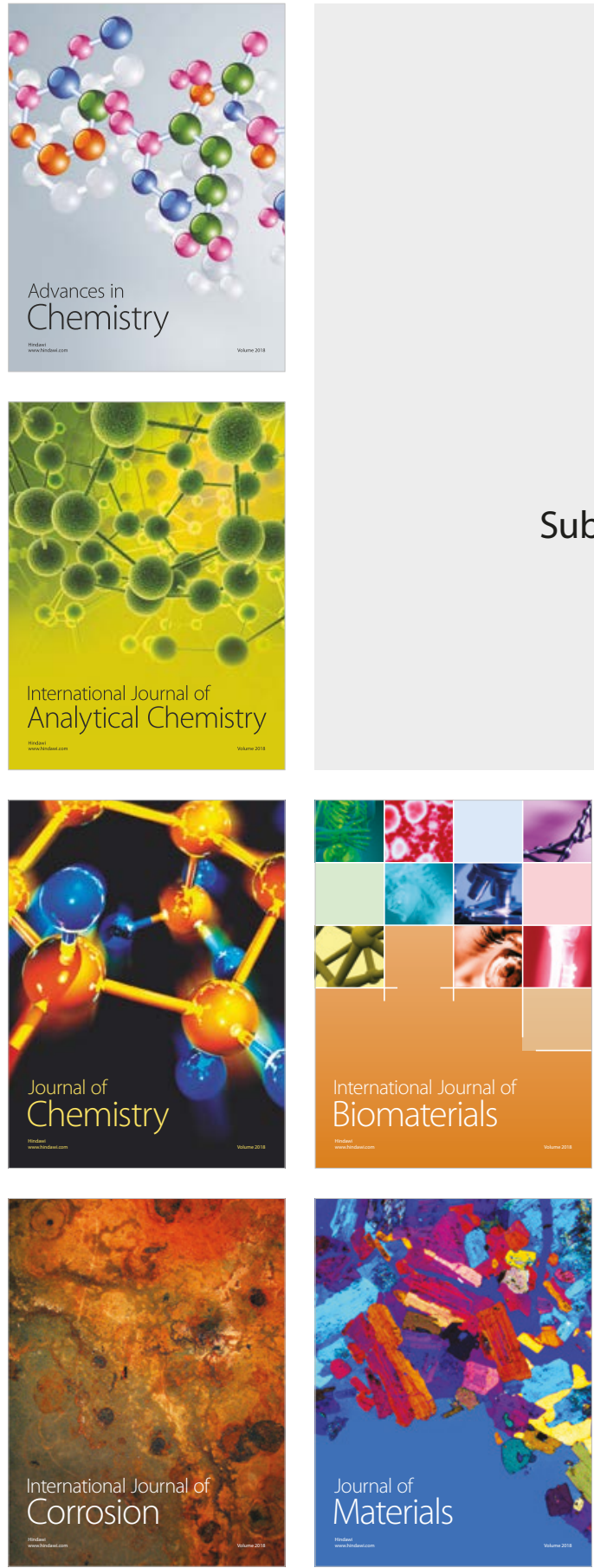

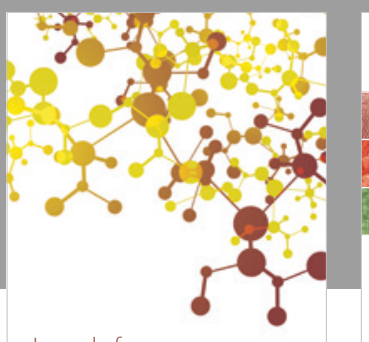

Journal of

Applied Chemistry
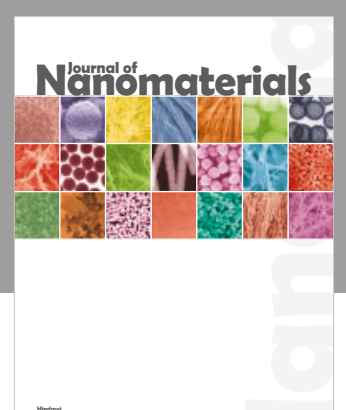

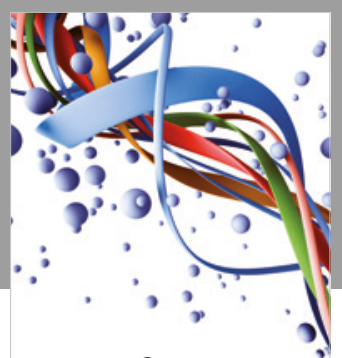

Scientifica

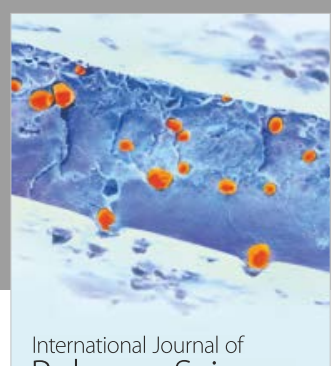

Polymer Science

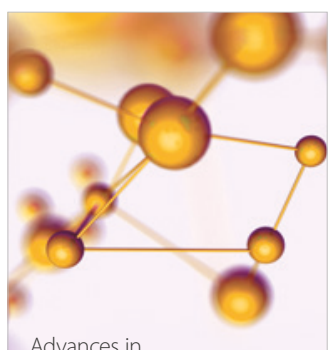

Physical Chemistry
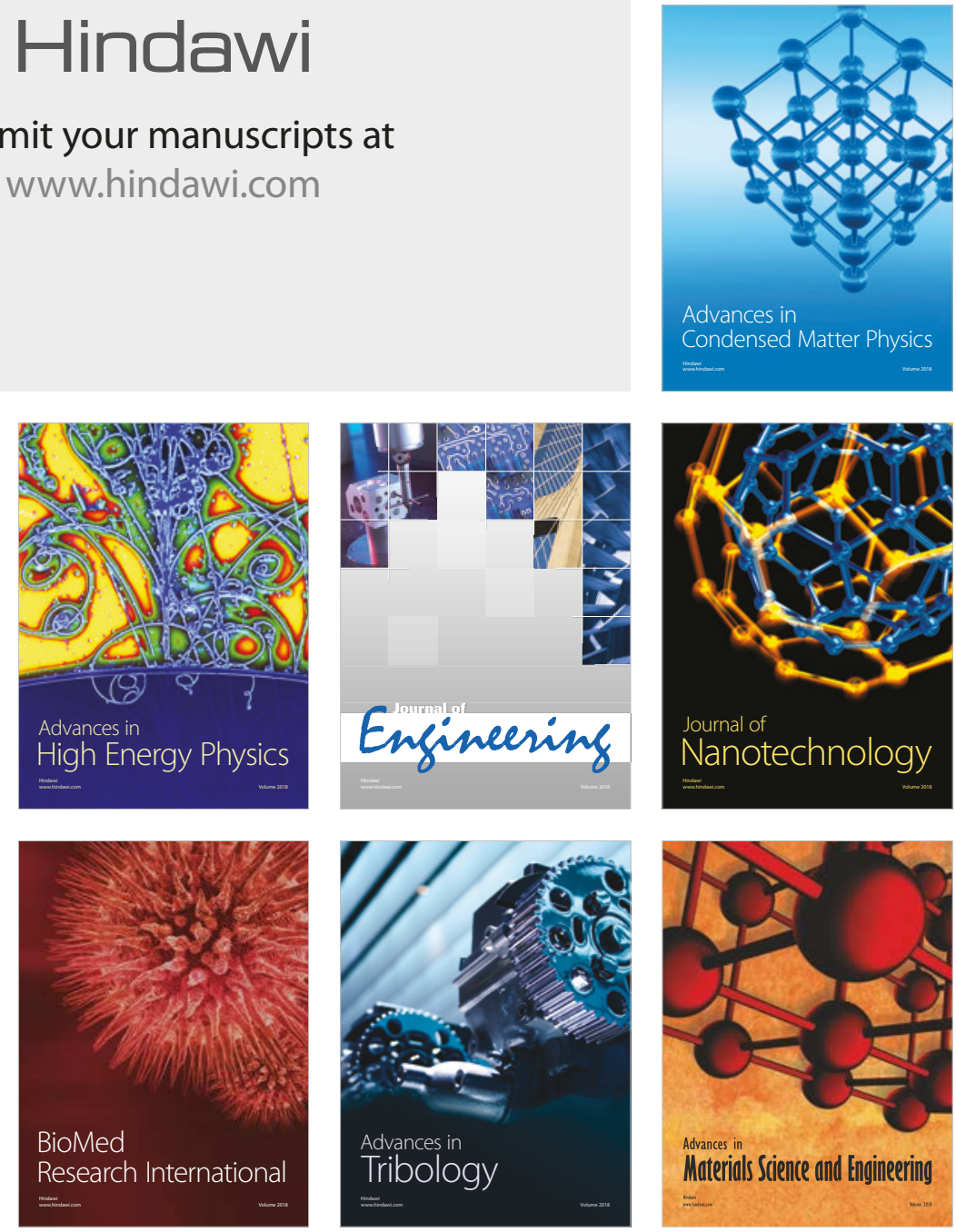